\title{
NIVEL SOCIOECONÓMICO BAJO Y EL DOLOR INCAPACITANTE EN PACIENTES DE LA UNIDAD DEL DOLOR
}

\author{
LOW SOCIOECONOMIC STATUS AND DISABLING PAIN \\ IN PATIENTS OF THE PAIN UNIT
}

\author{
Ana Isabel Masedo Gutierrez* \\ Laura Camacho Martel ${ }^{* *}$
}

\begin{abstract}
RESUMEN
Objetivo: Conocer la asociación entre el nivel socioeconómico que presentaban los pacientes diagnosticados de dolor crónico que acudían a la Unidad del Dolor y su adaptación al dolor crónico en términos de dolor, discapacidad y estado de ánimo. Material y método: Se realizó un estudio correlacional con una muestra de pacientes con dolor crónico que acudían a la Unidad del Dolor del Hospital Carlos Haya de Málaga. Mediante el uso de autoinformes que fueron administrados de forma oral, se midieron las siguientes variables: el nivel socioeconómico, el dolor, la discapacidad y el estado de ánimo. Se aplicó un ANOVA para comparar a los pacientes en función del nivel socioeconómico (NSE). Resultados: El grupo NSE bajo, formado mayoritariamente por mujeres $(85,7 \%)$, presentaban un dolor más incapacitante para las actividades de la vida diaria y consumían más medicación. Conclusión: Los resultados revelan que las condiciones socioeconómicas pueden suponer un factor de riesgo de una experiencia más desadaptativa del dolor crónico.
\end{abstract}

Palabras clave: Nivel socioeconómico, dolor crónico, personas con discapacidad, enfermería.

\begin{abstract}
Objective: To determine the association between the socioeconomic status of patients diagnosed with chronic pain entering the Pain Unit and their adaptation to chronic pain in terms of pain, disability and mood. Method: This is a cross-sectional study of 133 chronic pain patients attending the Pain Unit at the Carlos Haya Hospital in Málaga. By means of oral self-reports the following variables were measured: socioeconomic level, pain and discomfort, disability and mood. An ANOVA test was used to compare patients according to their Socioeconomic Status (SES). Results: The low SES group had more disabling pain for daily activities and took more medication. The low NSE group consisted mainly of women (85.7\%). Conclusion: The results reveal that socioeconomic conditions may be a risk factor for more disabling chronic pain.
\end{abstract}

Key words: Socioeconomic status, chronic pain, disabled persons, nursing.

Fecha recepción: 30/03/15 Fecha aceptación: 08/11/16

${ }^{*}$ PhD. Profesora del Departamento de Personalidad, Evaluación y Tratamientos Psicológicos. Facultad de Psicología. Universidad de Málaga, 29071. Málaga, España. E-mail: masedo@uma.es

${ }^{* *}$ PhD. Profesora asociada del Departamento Psicología Social, Trabajo Social, Antropología Social y Estudios de Asia Oriental. Universidad de Málaga. Málaga, España. E-mail: lcamacho@uma.es 


\section{INTRODUCCIÓN}

El dolor crónico es una condición altamente prevalente que tiene un fuerte impacto sobre la salud del individuo y sobre los costos que implican a múltiples dispositivos sociales (compañías de seguros, servicios de salud y servicios sociales, empresas, instituciones públicas, etc.) (1). Actualmente no hay lugar a duda de que el dolor requiere de una aproximación interdisciplinar en el que la enfermería, junto con otras áreas profesionales de la salud (medicina, terapia ocupacional, fisioterapia y psicología), tiene un papel esencial (2).

El estudio de los factores de riesgo de padecer dolor crónico ha crecido en los últimos diez años y el nivel o status socioeconómico ha sido considerado un factor de riesgo en múltiples casos estudiados. Un estudio epidemiológico de amplio espectro realizado en la región mediterránea de Cataluña en España informaba que la prevalencia de dolor crónico entre personas mayores (entre 65-85 años de edad) era mayor en mujeres. También había mayor prevalencia entre aquellas personas que tenían un menor nivel educativo $\mathrm{y}$, por último, señalaban una mayor prevalencia de dolor crónico entre aquellas personas con ocupaciones laborales de baja cualificación (3). Un segundo estudio epidemiológico del dolor crónico en España ha puesto en evidencia que una proporción significativa de pacientes con dolor crónico pertenecen a una clase social baja $(71,1 \%)$ y que en este grupo hay un número superior de mujeres $(60,7 \%)(4)$.

En la revisión de la literatura realizada para el presente trabajo, también se han identificado estudios internacionales que han prestado evidencia a la vulnerabilidad que supone proceder de un bajo nivel socioeconómico ante la experiencia de una condición asociada a dolor crónico. En un estudio longitudinal multicéntrico, los auto- res escogieron a pacientes que habían sufrido un trauma o una lesión que podía derivar en dolor crónico. Tres años después de la lesión o el trauma, el estatus socioeconómico bajo junto con la severidad del dolor y de la lesión fueron los principales predictores de desarrollar un dolor crónico, si bien el poder predictivo de dichos factores combinados fue calificado por los propios autores como modesto (5). Otro interesante trabajo empírico estudió a una muestra extensa de sujetos que habían sufrido un accidente de tráfico haciéndoles un seguimiento posterior hasta 1 año después del accidente. Se encontró que, entre estos sujetos, la condición de vivir en un barrio de bajo estatus socioeconómico incrementaba la posibilidad de tener mayor dolor musculoesquelético así como mayor interferencia del dolor con el desarrollo de actividades de la vida diaria (6).

Entre las personas ya diagnosticadas de dolor crónico algunos estudios han mostrado que hay una peor adaptación en términos de discapacidad, trastornos del estado de ánimo, entre otros indicadores. En Suecia tener un bajo y medio nivel educativo y nivel socioeconómico permitía predecir, mediante análisis de regresión, el diagnóstico de espondiloartritis y de dolor crónico (7). Recientemente, en una población de Singapur hallaron que en los sujetos clasificados en un nivel socioeconómico bajo, el dolor crónico se asociaba al desempleo y al deterioro funcional en términos de discapacidad (8). Green and Hart-Johnson (9) en los Estados Unidos, utilizando la escala Hospital Anxiety and Depression Scale (HADS), estudiaron las disparidades entre ser negro y blanco en relación al dolor crónico. Llegaron a la conclusión de que aquellas personas de raza negra que vivían en un barrio de bajo nivel socioeconómico presentaban mayor dolor, mayor discapacidad y peor estado de ánimo en términos de ansiedad y depresión.

El objetivo de este trabajo fue conocer la asociación entre el nivel socioeconómico que 
presentaban los pacientes diagnosticados de dolor crónico que acudían a la Unidad del dolor y su adaptación al dolor crónico en términos de dolor, discapacidad y estado de ánimo. Más concretamente, se estudiará si proceder de un nivel socioeconómico bajo se asocia a consecuencias más desadaptativas en las variables dependientes evaluadas: dolor informado, localización del dolor, discapacidad, consumo de medicamentos y trastornos del estado de ánimo.

\section{MATERIAL Y MÉTODO}

\section{Diseño}

Este es un estudio correlacional que trata de estimar si existen diferencias en función del nivel socioeconómico en las variables dependientes: diagnóstico, dolor informado, localización del dolor, estado de ánimo y discapacidad

\section{Participantes}

Todos los pacientes que acudían a recibir tratamiento por su dolor a la Unidad de Dolor de la Residencia Carlos Haya de Málaga. La muestra inicial se componía de 141 pacientes, sin embargo 8 fueron eliminados, ya que poseían un nivel socioeconómico alto y el número no era suficiente para establecer una categoría que permitiese ser comparada con las otras (NSE bajo y medio), siendo analizados finalmente 133 pacientes que estaban siendo tratados por un dolor que no remitía (con duración superior a 6 meses). Los participantes tenían una edad media de 54,5 años. El 29\% de los participantes se encontraba en un rango de edad comprendido entre los $24 \mathrm{y}$ los 45 años, el $26 \%$ tenían entre 45 y 55 años y el $43,1 \%$ se hallaba en el rango 56-80 años. La muestra de pacientes analizada contaba con 75 mujeres $(56,2 \%)$ y 58 hombres $(43,8 \%)$.

\section{Procedimiento}

A aquellos pacientes que habían sido atendidos por el médico se les ofrecía la posibilidad de participar voluntariamente en un estudio sobre los factores psicosociales que influían en el dolor. Todos los pacientes que aceptaron participar fueron entrevistados entre octubre de 2012 y junio de 2013 en una de las salas del servicio médico habilitada para ese fin.

Este estudio fue aprobado por el Comité Ético del Hospital Carlos Haya de Málaga y se solicitó a todos los pacientes el Consentimiento Informado. El Modelo de Consentimiento utilizado incluía las cláusulas: 1) que su participación era totalmente voluntaria y 2) que los datos que ellos proporcionaban eran anónimos y que serían utilizados únicamente con fines de investigación. Las entrevistas se realizaron sin acompañantes y los cuestionarios se contestaban de forma oral.

\section{Variables e instrumentos}

- Nivel socioeconómico (NSE): El cuadernillo de evaluación constaba de una serie de preguntas destinada a anotar la ocupación laboral de los pacientes que permitían determinar: a. El estatus laboral: trabajador en activo, jubilado o en paro; b. Si recibía o no pensión por su condición de invalidez; c. Ocupación. Se estableció una lista de categorías ocupacionales por sectores: servicios, construcción, agricultura, artesanía, educación, oficinista y profesión liberal. En función de estas preguntas, se establecieron tres categorías: Nivel Socioeconómico Bajo, Medio y Alto. En el grupo NSE bajo se agruparon a aquellos pacientes que no recibían ningún tipo de retribución (desempleados, amas de casa y jubilados sin pensión por invalidez). La categoría NSE medio se componía por trabajadores en activo en sectores de servicios, de la construcción, agrícola, oficinista y artesanía, amas de casa con pensión y jubilados con pensión por invalidez. La categoría 
NSE alto se componía por pacientes que eran funcionarios o profesiones liberales con dedicación activa. Esta categoría fue eliminada, por las razones señaladas previamente.

- Dolor y localización del dolor: Para evaluar el dolor informado se pedía a los pacientes que evaluaran mediante una escala de 0 a 10 (donde 0 es nada de dolor y 10 el peor dolor imaginable) la intensidad del dolor en la últimas dos semanas, diferenciando entre: el dolor más liviano, el dolor como media que había padecido en estas dos semanas, el peor dolor que había tenido y el dolor que sentía en ese momento (Ej.: Indique el número que describe la intensidad del dolor que ha tenido en las pasadas dos semanas como media). Así, para el estudio fue calculada la suma de las cuatro estimaciones del sujeto. La localización y generalización del dolor fue evaluada mediante la figura humana del Cuestionario de dolor McGill en su adaptación al castellano por los autores Lázaro et al. (10). En esta versión, los pacientes señalan la zona en que tienen dolor habitualmente. Posteriormente codificamos las respuestas entre zona cervical, torácica-dorsal, lumbar-renal, vertebral-lumbar, pierna y zona sacra. Yunus et al. (11) definían dolor generalizado cuando el dolor se daba al menos en 4 o más sitios anatómicos diferenciados por 3 meses.

Por último, se registró la medicación específica que se tomaba para el dolor. Si los participantes tomaban solamente antiinflamatorios no esteroideos (AINES) se especificaba 1, si tomaban AINES y coadyuvantes o opioides débiles se asignaba un 2 y si tomaba AINE, coadyuvantes y opioides débiles se especificaba 3 y finalmente se asignaba un 4 si se trataba con opioides fuertes (bomba de morfina).

- Estado de ánimo: Para estimar el estado de ánimo se utilizó la Hospital Anxiety and Depression Scale (12) adaptada por Quintana et al. (13). La escala está compuesta por 14 ítems que se responden mediante una escala Likert de 1 a 4 puntos, según el grado en que identifican su estado anímico actual con los ítems. El cuestionario cuenta con la posibilidad de obtener puntuaciones, una puntuación para la ansiedad (ítems 1, 3, 5, 7, 9, 11,13 ) y otra para depresión (ítems 2, 4, 6, $8,10,12,14)$. El rango de puntuaciones de las subescalas de ansiedad y depresión oscila entre 7 y 28 . El sentido de las puntuaciones es que a mayor puntuación puede interpretarse que el paciente presenta más ansiedad o más depresión. La versión española de la escala mostraba adecuada fiabilidad ( $\alpha$ : 0,86 para ansiedad; $\alpha: 0,86$ para depresión) (13).

- Discapacidad: Se utilizó el Roland-Morris Disability Questionnaire (RMDQ) (14) en su versión española (14) que cuenta con 24 ítems que evalúa las limitaciones que el dolor les ocasiona en la vida diaria. Los pacientes deben marcar con un SI en caso de que la frase se adecue a su realidad. Se obtiene una puntuación total que oscila entre 24 y 42; a mayor puntuación, mayor discapacidad. En la actualidad existen versiones en 18 idiomas, entre ellas la adaptada al castellano cuenta con dos versiones europeas y una canadiense. La utilizada para este estudio mostró una alta consistencia interna $(\alpha: 0,90)$, similar a los valores informados en otros estudios $(\alpha$ : $0,84,0,90$ y 0,93 ) para la misma escala (15).

\section{Análisis estadístico}

En primer lugar se obtuvo una descripción del sexo, la edad y los diagnósticos según el historial médico de los pacientes en cada uno de los grupos estudiados: Nivel socioeconómico bajo y Nivel socioeconómico medio y se usó la prueba no paramétrica $U$ de Mann-Whitney para contrastar que no había diferencias clínicas relevantes entre los dos grupos. En segundo lugar, se aplicó la prueba estadística ANOVA para comparar a los pacientes en función del nivel socioeconómico 
(NSE) medio y bajo en el dolor informado, la discapacidad y el estado de ánimo de los pacientes.

\section{RESULTADOS}

Como puede apreciarse en la Tabla 1, el $64,61 \%$ del total de los sujetos procedían de un NSE medio y el 35,39\% respondían a los criterios que establecimos para la categoría NSE bajo. En el grupo de nivel socioeconómico bajo había un alto porcentaje de mujeres $(85,7 \%)$, como puede apreciarse en la Tabla 1.

Los diagnósticos de los pacientes se agruparon de acuerdo a las siguientes categorías: dolor que afecta al raquis (dolor de patología lumbar y cervical) 39\% del total; dolor de naturaleza osteoarticular (poliartrosis, dolor óseo, etc.) 32,2\%; fibromialgia o dolor generalizado $12,8 \%$ y finalmente el dolor neuropático (neuralgias, postblasetomía, etc.) $17,4 \%$ del total de los pacientes. Se usó la Prueba U de Mann-Whitney para comparar las variables categóricas y no se apreciaron diferencias significativas entre los diagnósticos según el NSE.

Tabla 1: Número y porcentaje de hombres y mujeres en función del nivel socioeconómico (NSE) medio y alto.

\begin{tabular}{lcc}
\hline & NSE Medio & NSE Bajo \\
& $\mathrm{f}(\%)$ & $\mathrm{f}(\%)$ \\
\hline Hombres & $51(60,7 \%)$ & $7(14,3 \%)$ \\
Mujeres & $33(39,3 \%)$ & $42(85,7 \%)$ \\
\hline Total & $84(64,6 \%)$ & $49(35,4 \%)$ \\
\hline
\end{tabular}

En cuanto a la localización del dolor, el $21,9 \%$ de los pacientes tenían dolor solo en una localización, en dos el 17,5\%, en tres el $16,8 \%$, en cuatro el $11,7 \%$, en cinco el $8,8 \%$ y en más de cinco, el 6,6\% de los pacientes. En resumen, el 61,4\% de los pacientes tenían un dolor asociado a más de una localización corporal. No se apreciaron diferencias significativas en la localización del dolor en función del NSE (t: -.457 (gl 130); Sig. 649).

En cuanto a la medicación que tomaban los pacientes en el momento de la entrevista, un 24,1\% tomaban AINES solamente, 41,6\% tomaban AINES y coadyuvantes, el 15\% tomaban AINES y opioides débiles y el 23,4\% tomaban AINES, coadyuvantes y opioides débiles. Un 5\% tomaba opioides fuertes.
Se realizó ANOVA para comprobar si había diferencias significativas en función del NSE en las variables: dolor informado, ansiedad, depresión, discapacidad y consumo de medicamentos. Como se puede apreciar en la Tabla 2, la variable NSE se relacionó significativamente con cantidad de medicamentos, siendo superior en el grupo de NSE bajo que en el grupo NSE medio. El NSE también se relacionó significativamente con el grado de discapacidad de los sujetos, siendo más alta en el grupo de NSE bajo que en el grupo de NSE.

En cuanto al dolor informado se aprecian informes de dolor más altos en el grupo de NSE bajo, sin significancia estadística. Las variables depresión y ansiedad no mostraban diferencias significativas en función del NSE. 
Tabla 2. Diferencias en la cantidad de medicación, dolor informado, ansiedad, depresión y discapacidad en función del NSE medio y bajo.

\begin{tabular}{lcccc}
\hline Variables & $\begin{array}{c}\text { NSO Medio } \\
\text { Media (DT) }\end{array}$ & $\begin{array}{c}\text { NSO Bajo } \\
\text { Media (DT) }\end{array}$ & F & Valor p \\
\hline Dolor informado & $26.884(6.759)$ & $28.045(8.274)$ & 3.595 & 0,06 \\
Cantidad de medicación & $2.448(1.112)$ & $1.977(0.875)$ & 9.213 & 0,003 \\
Ansiedad & $17.359(4.837)$ & $15.613(5.021)$ & 0.344 & 0,559 \\
Depresión & $17.807(5.519)$ & $17.886(6.365)$ & 0,45 & 0,503 \\
Discapacidad & $34.551(6.249)$ & $37.250(6.816)$ & 10.061 & 0,002 \\
\hline
\end{tabular}

\section{DISCUSIÓN Y CONCLUSIÓN}

Los profesionales de la salud que más están en contacto con el dolor de los pacientes posiblemente sea el personal de enfermería y el presente estudio está destinado a subrayar la importancia de los factores sociales (en este caso socioeconómicos) en la experiencia de dolor con el fin de subrayar que el abordaje del mismo debe seguir siendo multidisciplinar, dada la naturaleza multidimensional del dolor (16).

El presente estudio proporciona evidencias que respaldan que los pacientes de dolor crónico que proceden de un nivel socioeconómico bajo consumen mayor cantidad de medicamentos y presentan mayor discapacidad para realizar las actividades de la vida diaria, ello es concordante con estudios recientes $(5,8)$.

En el grupo de NSE bajo hay un alto porcentaje de mujeres $(85,7 \%)$ y este dato es consonante con los encontrado en estudios previos (5). Futuros estudios deberían contrastar este dato con muestras más representativas y amplias en las comunidades andaluzas. Este dato contrasta con los datos ofrecidos de la zona mediterránea de Cataluña, donde se destacaba una alta prevalencia del dolor crónico en mujeres que se asociaba a una baja cualificación educativa y laboral (3). En el campo de estudio del dolor crónico no hay estudios destacados que contemplen la posible desigualdad social en relación a la mujer en el campo de investigación en torno al dolor crónico, sin embargo podría ser una línea de investigación fructífera.

Por otro lado, los diagnósticos principales eran de tipo músculo-esquelético y/o espinal y no se han detectado diferencias significativas en la generalización de la localización del dolor ni en los diagnósticos de los pacientes en función del NSE, así que, en principio, no puede concluirse que las diferencias encontradas en función del NSE en términos de discapacidad puedan deberse a la condición médica. Además, consistentemente con los datos que ofrecen estudios recientes de epidemiología del dolor crónico no maligno en España (4), se ha encontrado que un alto porcentaje de estos pacientes $(78,1 \%)$ tenían dolor en más de una zona corporal. En definitiva, se podría concluir que de acuerdo a lo estudiado el perfil de paciente que acude a la Unidad de Dolor con alta discapacidad, con dolor recurrente de alta intensidad, con un alto consumo de medicamentos, corresponde predominantemente al de una mujer de nivel socioeconómico bajo y que aproximadamente tiene 55 años de edad.

La escasez de recursos económicos en una persona que se enfrenta a una enfermedad de dolor crónico actuaría como un factor de vulnerabilidad y de riesgo para un dolor más incapacitante. Así, en este trabajo se confir- 
ma que, en el caso de las personas con dolor crónico, cuando los recursos socioeconómicos que posee son menores, la persona recurre a más medicina y es víctima de un mayor deterioro físico y funcional asociado al dolor, sin embrago las características metodológicas del presente estudio limitan para sostener una dirección causal entre estas variables. Los estudios previos realizados en este sentido de corte longitudinal, sí que han podido proporcionar evidencia a que los factores socioeconómicos son factor de riesgo para padecer dolor crónico más que consecuencias del dolor crónico $(5,7)$. No obstante, los resultados que aquí se presentan, al menos alientan a considerar en líneas futuras de estudio los condicionamientos económicos, sociales y laborales a los que está asociada la cronicidad del dolor.

Por otra parte, los resultados de este estudio no han revelado que los pacientes de NSE bajo presenten más problemas en el estado de ánimo. Cabe destacar que la ansiedad, la depresión y los trastornos afectivos en general de los pacientes con dolor crónico ( $y$ también el dolor y la discapacidad) han mostrado una relación robusta con otras variables psicológicas que no han sido analizadas en este trabajo. Ramírez-Maestre et al. (17) realizaron un amplio estudio multimuestra de 686 pacientes con dolor crónico muscoloesquelético en la región sur del mediterráneo y pusieron de manifiesto, entre otros resultados de enorme interés, que las variables que mejor predicen los problemas de estado de ánimo y el deterioro funcional de los pacientes de dolor crónico son la resiliencia, la aceptación del dolor y el miedo-evitación del dolor.

Los resultados aquí encontrados aportan evidencia a la conclusión de que las variables sociodemográficas de tipo económico y laboral tienen una influencia más bien en un plano corporal antes que afectivo ya que se relacionan con el deterioro funcional y la discapacidad, la medicación consumida y el dolor. Futuros trabajos deberían integrar el estudio de todos los agentes de vulnerabilidad y factores de riesgo asociados al dolor crónico con el fin de determinar el peso y la importancia de los factores socioeconómicos y las variables de la persona.

Por otra parte, queremos destacar que el tamaño de la muestra no es muy grande y que se compone de pacientes atendidos en un servicio médico muy específico como es la unidad de dolor. En este sentido, el alcance de los resultados del presente estudio cuenta con claras limitaciones y deberían ser tomados con cierta cautela a la hora de ser extrapolados a toda la población.

Creemos que esta línea de investigación tiene que ser más explorada ya que los estudios al respecto son sumamente escasos. No obstante, se reconocen limitaciones metodológicas en este trabajo. La muestra estudiada se restringe a aquellos pacientes que nos fueron remitidos por los médicos y no conocemos cuántos de los pacientes atendidos en la Unidad no accedieron a participar ni tampoco conocemos qué pacientes no nos fueron remitidos por los facultativos. Así mismo, el nivel socioeconómico se estimó mediante encuesta y a través del informe del sujeto. El diseño metodológico del presente estudio tiene limitaciones al ser de corte transversal. Futuros estudios podrían realizar estos contrastes con aproximaciones metodológicas cuasi experimentales.

\section{REFERENCIAS}

1. Torralba A, Miguel A, Darba J. Situación actual del dolor crónico en España: iniciativa "Pain Proposal". Rev Soc. Esp Dolor. 2014; 21(1): 16-22.

2. Miró J. Dolor crónico: procedimientos de evaluación e intervención psicológica. Bilbao: Desclee de Bower; 2003. 217 p.

3. Miró J, Paredes S, Rull M, Queral R, Miralles R, Nieto R, et al. Pain in older adults: A prevalence study in the Mediterranean 
region of Catalonia. Eur J Pain. 2007; 11(1): 83-92.

4. Soares K, Sola I, Aromataris E, Tornero J, Pérez C, Margarit C, et al. Epidemiology of chronic non-malignant pain in Spain. United Kingdom: Fundación Grünenthal; 2010. $202 \mathrm{p}$.

5. Holmes A, Williamson O, Hogg M, Arnold C, O'Donnell ML. Determinants of chronic pain 3 years after moderate or serious injury. Pain Med [Internet]. 2013 [citado 01 mar 2015]; 14(3): 336-44. Disponible en: http://onlinelibrary.wiley. com/wol1/doi/10.1111/pme.12034/full

6. Ulirsch JC, Weaver MA, Bortsov AV, Soward AC, Swor RA, Peak DA, et al. No man is an island: living in a disadvantaged neighborhood influences chronic pain development after motor vehicle collision. Pain. 2014; 155(10): 2116-23.

7. Jöud A, Petersson IF, Jordan KP, Löfvendahl S, Grahn B, Englund M. Socioeconomic status and the risk for being diagnosed with spondyloarthritis and chronic pain: a nested case-control study. Rheumatol. 2014 Int; 34(9): 1291-8.

8. Liang W, Sin D, Wen C, Zong L, Shibli S, Choon-Huat G. Chronic Pain in a Low Socioeconomic Status Population in Singapore: A Cross-Sectional Study. Pain Med [Internet]. 2016 [citado 01 mar 2015]; 17(5): 864-876. Disponible en: https://academic.oup.com/painmedicine/article/17/5/864/1752970/Chronic-Pain-in-a-Low-Socioeconomic-Status

9. Green CR, Hart-Johnson T. The association between race and neighborhood socioeconomic status in younger Black and White adults with chronic pain. J Pain. 2012; 13(2): 176-86.
10. Lázaro C, Bosh F, Torrubía R, Baños JE. The development of a Spanish questionnaire for assessing pain: Preliminary data concerning reliability and validity. Eur J Psychol Assess. 1994; 10(2): 145-151.

11. Yunus M, Kalyan-Raman UP. Muscle biopsy findings in primary fibromyalgia and other forms of nonarticular rheumatism. Rheum Dis Clin North Am. 1989; 15(1): 115-134.

12. Zigmond AS, Snaith RP. The hospital anxiety and depression scale. Acta Psychiatr Scand. 1983; 67(6): 361-79.

13. Quintana J M, Padierna A, Esteban C, Arostegui I, Bilbao A, Ruiz I. Evaluation of the psychometric characteristics of the Spanish version of the Hospital Anxiety and Depression Scale. Acta Psychiatr Scand. 2003; 107(3): 216-221.

14. Roland M, Morris R. A study of the natural history of back pain. Part I: development of a reliable and sensitive measure of disability in low-back pain. Spine. 1983; 8(2): 141-4.

15. Kovacs FM, Llobera J, del Real MT G, Abraira V, Gestoso M, Fernández C, et al. Validation of the spanish version of the Roland-Morris Questionnaire. Spine. 2002; 27(5): 538-542.

16. Melzack R, Wall PD. Pain mechanisms: a new theory. Science. 1965 Nov 19; 150(3699): 971-979.

17. Ramírez-Maestre C, Esteve R, López Martínez A. Fear-avoidance, pain acceptance and adjustment to chronic pain: a cross-sectional study on a sample of 686 patients with with chronic spinal pain. Ann Behav Med. 2014; 48: 402-410. 